\title{
IMMERSIONS OF POSITIVELY CURVED MANIFOLDS INTO MANIFOLDS WITH CURVATURE BOUNDED ABOVE
}

\author{
NADINE L. MENNINGA
}

\begin{abstract}
Let $M$ be a compact, connected, orientable Riemannian manifold of dimension $n-1 \geq 2$, and let $x$ be an isometric immersion of $M$ into an $n$ dimensional Riemannian manifold $N$. Let $K$ denote sectional curvature and $i$ denote the injectivity radius. Assume, for some constant positive constant $c$, that $K(N) \leq 1 /\left(4 c^{2}\right), 1 / c^{2} \leq K(M)$, and $\pi c \leq i(N)$. Then the radius of the smallest $N$-ball containing $x(M)$ is less than $\frac{1}{2} \pi c$ and $x$ is in fact an imbedding of $M$ into $N$, whose image bounds a convex body.
\end{abstract}

\section{INTRODUCTION}

We shall investigate the following questions. If a compact manifold $M_{1}$ with positive sectional curvature, is isometrically immersed in some ambient space $N$, what is the radius of the smallest ball in which its image lies? Additionally, when can the knowledge that the image lies inside a ball of restricted size be used to conclude that the immersion is an imbedding whose image bounds a convex body in $N$ ?

The question of when an immersion is in fact an imbedding whose image bounds a convex body was first considered by Hadamard. The most complete theory concerns immersions of nonnegatively curved hypersurfaces in Euclidean space. In this case, work of Hadamard [7], Stoker [13], and Chern and Lashof [6] culminated in the theorem of Sacksteder [10] and van Heijenoort [8]. According to this theorem, if $M$ is a complete, connected Riemannian manifold of dimension $n-1$ (throughout this paper we always assume that $n-1 \geq 2$ ) with $K(M) \geq 0$ and with at least one sectional curvature positive, and if $x: M \rightarrow R^{n}$ is an isometric immersion, then $x$ is an imbedding and $x(M)$ bounds a convex body. Do Carmo and Lima [4] and Wu [17] have developed alternative approaches to part of this material, and have obtained additional information about the images of the immersion.

Ambient spaces other than Euclidean space have also been considered. Do Carmo and Warner [5] showed that if the ambient space is a complete, simply connected space of constant curvature, $K$, and $M$ is complete and connected with $K(M) \geq K$, then any immersion $x: M \rightarrow N$ is an imbedding and $x(M)$ bounds a convex body.

Received by the editors August 22, 1988.

1980 Mathematics Subject Classification (1985 Revision). Primary 53C40; Secondary 53C20. 
We know of two theorems concerning the case where $N$ has variable curvature. Alexander has considered the case where the ambient manifold, $N$, is a Hadamard manifold; that is, where $N$ is complete and simply connected with nonpositive curvature [1]. Let $x$ be a hypersurface immersion of a compact, connected, orientable manifold $M$ of dimension $n-1$ into $N$, and assume the existence of a continuous unit normal field along $x$ with respect to which $x$ has a positive semidefinite second fundamental form. Again the conclusion is that $x$ imbeds $M$ into $N$ as the boundary of a convex body. Here the hypotheses concern the second fundamental form of the immersion rather than the sectional curvature of $M$; however, if $K(M)>0$ then the hypotheses are satisfied.

The case where $N$ has variable positive curvature was considered by Tribuzy [15]. There it was assumed that $N$ is complete, orientable, simply connected, noncompact, and satisfies $K \geq K(N)>0$ for some positive constant $K$. Let $x$ be a hypersurface immersion of a compact, connected, orientable manifold $M$ of dimension $n-1$ into $N$, and assume the existence of a continuous unit normal field along $x$ with respect to which $x$ has a second fundamental form whose eigenvalues are all at least $2 \sqrt{K_{0}}$. Then $x$ imbeds $M$ into $N$ as the boundary of a convex body. This hypothesis on the eigenvalues of the second fundamental form implies that $K(M)>4 K_{0}$.

The following theorem will be proved in $\S 3.2$.

Theorem 2. Let $M$ be a compact, connected, orientable Riemannian manifold of dimension $n-1$, with $n-1 \geq 2$, and $K(M) \geq 1 / c^{2}$, where $c$ is a positive constant. Let $N$ be an $n$-dimensional Riemannian manifold such that $\pi c \leq$ $i(N)$ and $K(N) \leq 1 /\left(4 c^{2}\right)$. Then any isometric immersion $x: M \rightarrow N$ imbeds $M$ into $N$ as the boundary of a convex body.

In the above theorem $i(N)$ is the injectivity radius of $N$. This theorem includes the theorems of Hadamard, Tribuzy, and Alexander in the case $K(M)>$ 0 . If $N$ is a simply connected space of constant curvature $K$, then the theorem of do Carmo and Warner is stronger than ours, in that they only require $K(M) \geq K$ whereas we have $K(M) \geq 4 K$. Note that in Theorem 2 the curvature of $N$ is allowed to be both positive and negative, whereas in all previous cases $K(N)$ was restricted to be either positive or nonpositive. Furthermore, rather than assuming $N$ to be simply connected, we have placed a certain lower bound on the injectivity radius $i(N)$ of $N$. For example, real projective spaces of curvature less than or equal to $1 /\left(4 c^{2}\right)$ satisfy the conditions of Theorem 2 .

The proof of Theorem 2 relies on the fact that $x(M)$ may be shown to lie in a ball with radius less than $\frac{1}{2} \pi c$. Spruck [12] showed that the smallest Euclidean ball in $R^{n}$ containing a compact, connected Riemannian manifold $M$ of dimension $n-1$ with $K(M) \geq 1 / c^{2}$ has radius $r<\frac{1}{2} \pi c$ and this bound is the best possible. The following theorem is a generalization of Spruck's result.

Theorem 1. Let $M$ be a compact, connected Riemannian manifold of dimension 
$m, m \geq 2$, with $K(M) \geq 1 / c^{2}$, where $c$ is a positive constant. Let $N$ be an $n$ dimensional Riemannian manifold such that $\pi c \leq i(N)$ and $K(N) \leq 1 /\left(4 c^{2}\right)$. If $x: M \rightarrow N$ is an isometric immersion, then $x(M)$ is contained in a metric ball of $N$ with radius $R<\frac{1}{2} \pi c$.

The bound in Theorem 1 is the best possible, in that for any $\varepsilon>0$ and simply connected space $N$ of constant curvature less than or equal to $1 /\left(4 c^{2}\right)$, there exist an $M$ satisfying the above conditions and an immersion $x: M \rightarrow N$ such that $x(M)$ lies in no ball of radius $\frac{1}{2} \pi c-\varepsilon$.

The fact that the bound on $R$ does not change with the curvature of $N$ may seem surprising at first, since the extrinsic radius of an immersed sphere of intrinsic curvature $1 / c^{2}$ does change as the curvature of the ambient space changes.

The conditions on $M$ in Theorem 1 are less restrictive than those of Theorem 2, where $M$ is both orientable and of codimension 1. In Theorem 1, $M$ need not be orientable and can be of any codimension.

This paper is based on the author's doctoral thesis presented at the University of Illinois. The author thanks her advisor, Professor Stephanie Alexander, for the valuable criticism she has given during the preparation of the thesis.

\section{The RAdius of THE SMALlest Ball CONTAINING A COMPACT MANIFOLD OF POSITIVE CURVATURE}

2.1. Background material. All manifolds are assumed to be $C^{\infty}$. We use the term immersion to mean immersion. All balls are assumed to be closed unless otherwise described. The convention that $1 / d^{2}=0$ when $d=\infty$ will be used throughout. Thus, the phrase "a sphere of curvature $1 / d^{2}$ " includes the Euclidean case unless it is specifically excluded.

A convex set $X$ in a complete Riemannian manifold will be one in which every two points of $X$ are joined by a minimizing geodesic which lies in $X$ and is unique in $X$. The closed metric ball $B(x, r)$ of radius $r$ at $x$ is called strongly convex if every two points, $p$ and $q$, in $B(x, r)$ are joined by a unique minimizing geodesic, $p q$, and the interior of $p q$ (the open arc) lies in the interior of $B(x, r)$. We assume $p q$ is parametrized by arclength and let $\overline{p q}$ represent the vector tangent to $p q$ with length equal to the length of $p q$. The radius of convexity at $p$ is the largest $r_{0}$ such that for all $r<r_{0}$ the closed ball $B(p, r)$ is strongly convex.

Bonnet's result that the diameter, $d(M)$, of a compact manifold $M$ of curvature at least $1 / c^{2}$ satisfies $d(M) \leq \pi c$ will be referred to as Bonnet's Theorem [3, p. 27]. Similarly, Toponogov's result that the length of a geodesic triangle on a manifold of curvature at least $1 / c^{2}$ does not exceed $2 \pi c$ will be referred to as Toponogov's Theorem [14, p. 326]. A geodesic triangle is a union of three minimizing geodesics.

2.2. The smallest ball containing a compact set. If $X$ is a compact subset of a complete Riemannian manifold, it is easy to see that there exists a closed ball of 
smallest radius $r$ containing $X$. In general there may not be a unique smallest ball; consider the equator of a sphere. On a right cylinder, a latitudinal circle is contained in an infinite number of smallest balls, one centered at each point on the circle. In the last example the smallest balls are not normal coordinate balls.

Let $N$ be a Riemannian manifold and $B(p, r)$ be a closed normal coordinate ball at $p$. We say a set $X \subset B(p, r)$ supports $B(p, r)$ if for each $v$ in $N_{p}$ the exponentiated hemisphere $\left\{\exp _{p}(u): u \cdot v \geq 0,|u|=r\right\}$ contains a point of $X$.

Lemma 1. Let $N$ be a complete Riemannian manifold and $X$ a compact subset of $N$. Let $B(p, R)$ be a closed ball of smallest radius containing $X$. If $B(p, R)$ is a normal coordinate ball then $X$ supports $B(p, R)$.

Proof. Suppose that $X$ does not support $B=B(p, R)$. Let $v \in N_{p}$ be a direction for which there is no support; i.e., suppose the set $\left\{\exp _{p}(u): u \cdot v \geq 0\right.$, $|u|=R\}$ contains no points of $X$. Since $X$ and $\partial B$ are compact, $X \cap \partial B$ is compact and there is a smallest closed and truncated solid cone $C$ in $N_{p}$ centered about $-v$ such that $\exp _{p} C$ contains $X \cap \partial B$. Thus, $C$ is the set of vectors $\left\{u \in N_{p}:|u| \leq R\right.$ and $\left.u \cdot v \leq a\right\}$ for some $a<0$. Let $\gamma$ be the geodesic starting at $p$ with direction $-v$. It is straightforward using the first variation equation to show that $\gamma(s)$, for small $s$, is the center of a ball containing $X$ and having radius $r$ less than $R$.

The ball $B=B(p, R)$ in the statement of Lemma 1 is guaranteed to be unique under certain conditions. In the following proposition, the assumption that $R$ be strictly less than the radius of convexity cannot be relaxed, as the example of the equator of a sphere shows.

Proposition 1. Let $N$ be a complete Riemannian manifold and $X$ a compact subset of $N$. Let $B(p, R)$ be a closed ball of smallest radius containing $X$. If $B(p, R)$ is a normal coordinate ball and $R$ is less than the radius of convexity at every $x$ in $X$, then $B(p, R)$ is the unique ball of smallest radius containing $X$.

Proof. Suppose $B(q, R)$ also contains $X$, where $q \neq p$. Let $\gamma$ be a geodesic ray from $p$ through $q$ and $p q$ the correpsonding segment. Let $x_{0}$ be the point of $X$ on the exponentiated hemisphere associated with $-\gamma$. Then $d\left(p, x_{0}\right)=R$ and $d\left(q, x_{0}\right) \leq R$. Thus $p q$ lies in the strongly convex ball $B\left(x_{0}, R\right)$. Note that $B\left(x_{0}, R\right)$ lies in a larger strongly convex ball and thus in an open normal coordinate ball. Now for $-\delta<s<\delta$, there is a smooth variation of geodesics $\alpha(s, t)$ for which $\alpha\left(s_{0}, t\right)$ is a unit speed geodesic from $\gamma\left(s_{0}\right)$ to $x_{0}$. Then at $p$

$$
\frac{d L(\alpha)}{d s}=-\left|\overline{p x}_{0}\right|^{-1}\left(\left\langle\overline{p q}, \overline{p x}_{0}\right\rangle\right) .
$$

Therefore, the angle between $p q$ and $p x_{0}$ is not obtuse since if it were, then 
$d L(\alpha) / d s$ would be positive at $p$ and $\left|\overline{\gamma(s) x_{0}}\right|$ would exceed $R$ for small positive $s$.

By construction the angle $x_{0} p q$ is not acute and hence it is a right angle. This is impossible since the interior of $p q$ lies in the interior of $B\left(x_{0}, R\right)$. Indeed, if the angle $x_{0} p q$ were a right angle there would be a geodesic segment $p z$ near $p q$, lying in the interior of $B\left(x_{0}, R\right)$ except for $p$, such that the angle $x_{0} p z$ is obtuse. As we have already seen, by the first variation formula, no such segment exists, and so the proof is complete.

2.3. Triangles on a sphere. We shall use the following corollary to the Rauch Comparison Theorem. A proof may be found in [3, p. 30].

Proposition 2. Let $N$ be a Riemannian manifold of dimension $n(n>2)$ and $K(N) \leq 1 / d^{2}$. Let $S^{n}$ be an $n$-sphcre of curvature $1 / d^{2}$. Assume that $I: N_{p} \rightarrow$ $S_{q}^{n}$ is a linear injection preserving inner products. If $r<\min \{i(N), \pi d\}$, then for any curve $\alpha$ in the closed ball $B(p, r)$ in $N$, we have

$$
L(\alpha) \geq L\left(\exp _{q} \circ I \circ\left(\exp _{p}\right)^{-1}(\alpha)\right) .
$$

Some of our comparisons will be based on the following fact from spherical trigonometry.

Lemma 2. Let $S^{n}$ be a sphere of dimension $n(n \geq 2)$ and constant curvature $1 / d^{2}, d<\infty$. Let $p_{1}$ and $p_{2}$ be points of distance $R$ from a third point $p$, where $r \leq \frac{1}{2} \pi d$. Then

$$
d\left(p_{1}, p_{2}\right)=2 d \cdot \sin ^{-1}\left(\sin (R / d) \sin \left(\frac{1}{2} \theta\right)\right)
$$

where $\theta$ is the measure of the angle between $p p_{1}$ and $p p_{2}$.

This formula corresponds to the formula

$$
d\left(p_{1}, p_{2}\right)=2 R \sin \left(\frac{1}{2} \theta\right)
$$

in the Euclidean case.

Let $B(p, R)$ be a smallest ball in $N$ containing the compact set $X$. We shall need a lower bound for the maximum length of a geodesic triangle formed with vertices in $X \cap \partial B(p, R)$. In the Euclidean case, the bound was computed by Spruck [12]. Lemma 3, which follows, gives the bound in the case where $N$ is a sphere of constant curvature $1 / d^{2}$.

Lemma 3. Let $N$ be a sphere of constant curvature $1 / d^{2}$ and $X$ be a compact set in $N$. Let $B(p, R)$ be a ball of smallest radius in $N$ containing $X$. Then if $R \leq \frac{1}{2} \pi d$, there is a geodesic triangle whose vertices are points of $X$ and whose length is at least the minimum of $4 R$ and $6 d \cdot \sin ((1 / \sqrt{2}) \sin (R / d))$. If $d=\infty$, then there exists a geodesic triangle whose vertices are points of $X$ and whose length is at least $4 R$.

Proof. Let $B=B(p, R)$. Suppose $d<\infty$. By Lemma 1, we know that $X$ supports $B$. Choose $p_{1}$ and $p_{2}$ in $X \cap \partial B$ so that $d\left(p_{1}, p_{2}\right)$ equals the 
maximal distance between any two points of $X \cap \partial B$. Let $\theta$ be the measure of angle $p_{1} p p_{2}$. Because $X$ supports $B$ we have $\frac{1}{2} \pi<\theta \leq \pi$.

If $\frac{1}{2} \pi \leq \theta<\pi$, let $v$ be the unit bisector in $N_{p}$ of the angle determined by $\overline{p p}_{1}$ and $\overline{p p}_{2}$. Let

$$
Y=\left\{w \in N_{p}:|w|=R \text { and } w \cdot v \leq 0\right\} .
$$

The exponentiated hemisphere, $\exp _{p} Y$, must contain at least one point $p_{3}$ of $X \cap \partial B$. Let $p_{4}=\exp _{p}(R v)$. If $\left(\overline{p p}_{3}, v\right)<0$, then there is a point $p_{5}$ of $\partial B$ such that $\left(\overline{p p}_{5}, v\right)=0$ and $p p_{5}$ is interior to the angle of $p_{3} p p_{4}$. Let $p_{5}=p_{3}$ if $\left\langle\overline{p p}_{3}, v\right\rangle=0$. Thus we have $L\left(p_{1} p_{2} p_{5}\right) \leq L\left(p_{1} p_{2} p_{3}\right)$ where $L\left(p_{i} p_{j} p_{k}\right)$ is the length of triangle $p_{i} p_{j} p_{k}$. We shall minimize the length $L=L\left(p_{1} p_{2} p_{5}\right)$.

Let $\beta$ be the measure of the angle $p_{1} p p_{5}$. By symmetry the measure of the angle formed by $p p_{2}$ and the ray opposite $p p_{5}$ is also $\beta$. Thus the measure of the angle $p_{2} p p_{5}$ is $\pi-\beta$. From Lemma 2 we have

$$
\begin{aligned}
L=2 d\left[\sin ^{-1}\left(\sin (R / d) \cdot \sin \left(\frac{1}{2} \theta\right)\right)+\sin ^{-1}\left(\sin (R / d) \cdot \sin \left(\frac{1}{2} \beta\right)\right)\right. \\
\left.+\sin ^{-1}\left(\sin (R / d) \cdot \cos \left(\frac{1}{2} \beta\right)\right)\right] .
\end{aligned}
$$

By the choice of $p_{1}$ and $p_{2}$, both $\beta$ and $\pi-\beta$ are less than $\theta$ and hence $\pi-\theta \leq \beta \leq \theta$. For a fixed $\theta, L$ is minimized at an extreme value for $\beta$. Indeed, $L$ is a concave function of $\theta$ and symmetric in $\beta$ about $\frac{1}{2} \pi$.

At an extreme value of $\beta$,

$$
L=2 d\left[2 \sin ^{-1}\left(\sin (R / d) \cdot \sin \left(\frac{1}{2} \theta\right)\right)+\sin ^{-1}\left(\sin (R / d) \cdot \cos \left(\frac{1}{2} \theta\right)\right)\right] .
$$

This expression of $L$ is minimized at one of the extreme values of $\theta$ since $L$ is a concave function of $\theta$. Therefore $L$ is minimized as $\theta$ approaches $\frac{1}{2} \pi$ or $\pi$. In the first case the expression becomes

$$
L=6 d\left[\sin ^{-1}((1 / \sqrt{2}) \sin (R / d))\right]
$$

and in the second, $L=4 R$.

2.4. Proof of Theorem 1. By Bonnet's theorem, any two points of $M$ can be joined by an $M$-geodesic of length less than or equal to $\pi c$. By a theorem of Synge [2, p. 194], in order that $x \circ \gamma_{s}$ be a geodesic of $N$, for a curve $\gamma$ of $M$, we must have $K(M)(P) \leq K(N)\left(X_{*}(P)\right)$ for any plane section $P$ of $M$ tangent to $\gamma$. Since $K(N)$ is known to be less than $K(M)$ for all plane sections $P$ and since $M$ is complete, we can conclude that $d\left(q_{1}, q_{2}\right)$ strictly exceeds $d\left(x\left(q_{1}\right), x\left(q_{2}\right)\right)$ for any $q_{1}$ and $q_{2}$ in $M$. It follows that any two points of $x(M)$ can be joined by an $N$-geodesic of length less than $\pi c$. Thus $x(M)$ is contained in an $N$-ball of radius less than $\pi c$.

Let $B=B(p, R)$ be an $N$-ball of smallest radius containing $x(M)$; in particular, $R<\pi c$. By Lemma 1, it follows that $x(M)$ supports $B$. Let $d=2 c$. Then $R<\pi c=\frac{1}{2} \pi d$.

Let $S^{n}$ be the $n$-sphere of curvature $1 / d^{2}$. Let $q \in S^{n}$ and let $I: N_{p} \rightarrow S_{q}^{n}$ be an inner product preserving map. Define the map $\varphi: B \rightarrow S^{n}$ by $\varphi(z)=$ 
$\exp _{q} \circ I \circ\left(\exp _{p}\right)^{-1}(z)$. This map takes a metric sphere of radius $r \leq R$ at $p$ to a metric sphere of radius $r$ at $q$, and an angle with measure $\theta$ at $p$ to an angle of measure $\theta$ at $q$. Since $x(M)$ supports $B, \varphi \circ x(M)$ supports $\varphi(B)$. By Lemma 3, there are three points, $q_{1}, q_{2}, q_{3}$, in $\varphi \circ x(M) \cap \partial \varphi(B)$ such that the geodesic triangle in $\varphi(B)$ with $q_{1}, q_{2}, q_{3}$ as vertices has length $L$, where

$$
L \geq \min \left\{4 R, 6 d \cdot \sin ^{-1}((1 / \sqrt{2}) \sin (R / d))\right\} .
$$

Let $p_{1}, p_{2}, p_{3}$ be points in $M$ such that $\varphi \circ x\left(p_{i}\right)=q_{i}$. Let $L_{M}\left(p_{1} p_{2} p_{3}\right)$ be the length of a geodesic triangle in $M$ with vertices $p_{1}, p_{2}, p_{3}$; similarly, $L_{S}$ will refer to the image triangle in $S$. Then

$$
L_{M}\left(p_{1} p_{2} p_{3}\right) \geq L_{S}\left(q_{1} q_{2} q_{3}\right) \geq \min \left\{4 R, 6 d \cdot \sin ^{-1}((1 / \sqrt{2}) \sin (R / d))\right\} .
$$

The first inequality follows from Proposition 2. have

Now, Toponogov's theorem gives $2 \pi c \geq L_{M}\left(p_{1} p_{2} p_{3}\right)$. Thus if $R \leq \frac{1}{4} \pi d$, we

$$
4 R=\min \left\{4 R, 6 d \cdot \sin ^{-1}((1 / \sqrt{2}) \sin (R / d))\right\}<2 \pi c
$$

or $R<\frac{1}{2} \pi c$. Note that if $\frac{1}{4} \pi d<R \leq \frac{1}{2} \pi d$, we have

$$
\sin (\pi / 6)<(1 / \sqrt{2}) \sin (R / d)
$$

and thus

$$
\pi d<6 d \cdot \sin ^{-1}((1 / \sqrt{2}) \sin (R / d)) .
$$

However, $\frac{1}{4} \pi d<R \leq \frac{1}{2} \pi d$ also gives

$$
6 d \cdot \sin ^{-1}((1 / \sqrt{2}) \sin (R / d)) \leq 2 \pi c=\pi d,
$$

which is a contradiction. Thus $R \leq \frac{1}{4} \pi d$ holds and hence also $R \leq \frac{1}{2} \pi c$.

Indeed, $R<\frac{1}{2} \pi c$, since $R=\frac{1}{2} \pi c$ could not occur when the minimum of $4 R$ is achieved; that is, if $\theta=\pi$ in the proof of Lemma 3. This would mean that two points of $x(M)$ were antipodal on $\partial B(p, R)$, and hence could be joined by an $N$-geodesic of length $\pi c$. But we know that any two points of $x(M)$ can be joined by an $N$-geodesic of length less than $\pi c$. Thus, $R=\frac{1}{2} \pi c$ would contradict $i(N) \geq \pi c$. This completes the proof of Theorem 1 .

\section{IMBEDDING AND CONVEXITY}

3.1. Background material. A Jacobi field $J$ along a geodesic $\gamma(s)$ is called normal if $J$ is everywhere normal to $\gamma$. Let $D / d s$ represent the covariant derivative in $N$ along a given curve. The second fundamental form $S$ of a hypersurface $M$ with respect to a given unit normal field $Z$ is given by $S_{p}(V, W)=\langle(D / d s(Z))(0), W\rangle$, where $V$ and $W$ are vectors tangent to $M$ at $p$ and $\gamma$ is a curve in $M$ with $\gamma^{\prime}(0)=Z_{p}$.

Suppose $\gamma$ is normal to a hypersurface $M$ at $\gamma(0)$. Then a Jacobi field $J$ along $\gamma$ is called an $M$-Jacobi field if $J(0)$ is tangent to $M$ and

$$
S_{\gamma(0)}(J(0), J(0))-D J \mid d s(0)
$$


is normal to $M$ at $\gamma(0)$, where $S$ is the second fundamental form of the surface $M$ corresponding to the normal $\gamma^{\prime}(0)[16$, p. 350]. A focal point of $M$ is a point of $\gamma$ at which a nontrivial $M$-Jacobi field along $\gamma$ vanishes.

In the proof of Theorem 2 we shall form a family of immersions by moving along the geodesics normal to a given immersion $x$. Using properties of this family of immersions to contradict the assumption that $x$ has self-intersections, we conclude that $x$ is an imbedding. We need the following result of Warner on focal points [16, p. 351].

Proposition 3. Let $M$ be a hypersurface on $N$, and let $\gamma$ be a geodesic normal to $M$ and parametrized by arclength. Let $a$ and $b, b>0$, be real numbers and let $t_{0}$ be the smallest positive solution to

$$
\cot \left(b^{1 / 2} t\right)=-a / b^{1 / 2}
$$

Let $S_{\gamma(0)}$ be the second fundamental form of $M$ with respect to $\gamma^{\prime}(0)$. Assume all the eigenvalues of $S_{\gamma(0)}$ are greater than or equal to $a$ and all the sectional curvatures of sectons of $N$ which are tangent to $\gamma$ are less than or equal to $b$. Then there are no focal points of $M$ on $\gamma \mid\left[0, t_{0}\right)$.

Our proof also makes use of Jacobi field estimates which are an immediate consequence of Corollary 2A in Karcher's paper [9]. We have the following lemma.

Lemma 4. Let $N$ be a complete Riemannian manifold with $K(N) \leq 1 / d^{2}(d<$ $\infty)$. Let $J(s)$ be a normal Jaocbi field along a geodesic $\gamma(s)$, with $|J|(0)=1$ and $|J|^{\prime}(0)=\lambda>0$. Then

$$
\begin{gathered}
|J|(S) \geq \cos (s / d)+d \lambda \sin (s / d), \\
|J|^{\prime}(s) \geq \lambda \cos (s / d)-(1 / d) \sin (s / d)
\end{gathered}
$$

for $0 \leq s \leq \frac{1}{2} \pi d$.

3.2. Proof of Theorem 2. The main point of this proof is to show that the immersion $x$ is in fact an imbedding. By Theorem $1, x(M)$ lies strictly inside a ball $B=B(p, R)$ with $R<\frac{1}{2} \pi c$. Choose $\varepsilon_{1}$ so that $R<\frac{1}{2}\left(\pi c-\varepsilon_{1}\right)$, and $d$ in $(0, \infty)$ so that $1 / d^{2} \leq 1 /\left(4 c^{2}\right)$ and $K(N)$ does not exceed $1 / d^{2}$ on $B\left(p,\left(\frac{3}{2}\right) \pi c\right)$. There is a continuous unit normal field $Z$ along the map $x$ (since $x(M)$ lies in an open normal coordinate ball). Since $1 / c^{2}-1 / d^{2}>0$, $Z$ may be chosen so that the second fundamental form $S$ of $x$ with respect to $Z$ is positive definite. Let

$$
\gamma(m, s)=\exp _{x(m)} s Z(m) .
$$

Thus for each fixed $m$ in $M$, there is a normal geodesic $\gamma_{m}$ at $x(m)=\gamma(m, 0)$ given by $\gamma_{m}(s)=\gamma(m, s)$. It follows from Proposition 3, with $a=0$ and $b=1 / d^{2}$, that if $0 \leq s<\frac{1}{2} \pi d$ and $\gamma_{m} \mid[0, s]$ lies in $B\left(p,\left(\frac{3}{2}\right) \pi c\right)$, then there is no focal point of $M$ along $\gamma_{m} \mid[0, s]$. Since $2 c \leq d$, there is no focal point if $0 \leq s \leq \pi c-\varepsilon_{1}$. 
Since $B$ lies in the open normal coordinate ball of radius $\pi c-\varepsilon_{1}$ at $x(M)$ for each $m$ in $M$, there is a first point $g(m)$ where $\gamma_{m}$ strikes $\partial B$. Furthermore, $g(m)$ is not a focal point of $M$ along $\gamma_{m}$.

Since $R<\frac{1}{2} \min \{\pi d, i(N)\}$, it can be shown that $B(p, R)$ is strongly convex. This statement is a consequence of the proof of Theorem 5.14 in Cheeger and Ebin [3, p. 103]. It follows that $\gamma_{m}$ cannot be tangent to $\partial B$ at the point $g(m)$; the argument is the same as that given in Proposition 1. Thus $\gamma_{m}$ strikes $\partial B$ transversely, and the map $g: M \rightarrow \partial B$ is a local diffeomorphism. Since $\partial B$ is diffeomorphic to an $(n-1)$-sphere the map $g$ is a diffeomorphism and hence $M$ is diffeomorphic to an $(n-1)$-sphere.

By the compactness of $M$ there exists an $\varepsilon_{2}$ such that there are no focal points of $M$ along any normal geodesic for $-\varepsilon_{2}<s<\pi c-\varepsilon_{1}+\varepsilon_{2}$. Thus for each fixed $s$ with $-\varepsilon_{2}<s<\pi c-\varepsilon_{1}+\varepsilon_{2}$, the mapping $x_{s}: M \rightarrow N$ given by $x_{s}(m)=\gamma(m, s)$ is an immersion.

Note that, along $\gamma_{m}$, each $M$-Jacobi field with respect to the immersion $x$ is also an $M$-Jacobi field with respect to the immersion $x_{s}$. We shall write

$$
S(V(s), V(s))=\frac{1}{2} D \mid d s\langle V(s), V(s)\rangle,
$$

where $V$ is any such $M$-Jacobi field along $\gamma_{m}$. For each $s$, the second fundamental form $S_{s}$ of $x_{s}$ with respect to $\gamma_{m}^{\prime}(s)$ agrees with $S$ in the sense that

$$
S_{s}(V(s), V(s))=S(V(s), V(s)) .
$$

Suppose $x$ is not an imbedding of $M$ into $N$. Then $x\left(q_{1}\right)=x\left(q_{2}\right)$ for distinct $q_{1}$ and $q_{2}$ in $M$. Since $R<\frac{1}{2} \pi c-\varepsilon_{1}$, the interior of $B\left(x(m), \pi c-\varepsilon_{1}\right)$ contains $x(M)$. Therefore, $\gamma\left(m, \pi c-\varepsilon_{1}\right) \notin x(M)$ for all $m$ in $M$. Thus there exists $t, 0 \leq t<\pi c-\varepsilon_{1}$, such that $x_{t}(M) \cap x(M) \neq \varnothing$ and $x_{s}(M) \cap x(M)=\varnothing$ for $t<s \leq \pi c-\varepsilon_{1}$. Hence, there exist distinct points $y$ and $z$ such that $x(z)=x_{t}(y)$. Furthermore, by the maximality of $t, x$ and $x_{t}$ are tangent at these two points. Thus we have

$$
Z_{t}(y)= \pm Z(z),
$$

where $Z_{t}(y)$ is $\gamma_{1}^{\prime}(t)$, a normal to $x_{t}$ at $\gamma(y, t)$. The choice $Z_{t}(y)=Z(z)$ contradicts the fact that $g$ is a diffeomorphism, so $Z_{t}(y)=-Z(z)$. It follows that both $x(y)=x_{t}(z)$ and $x(z)=x_{t}(y)$ hold (see Figure 1).

Let $\lambda_{1}$ be the maximum eigenvalue of the second fundamental form $S_{0}$ of $x$ at $y$ and $\lambda_{2}$ be the maximum eigenvalue of $S_{0}$ at $z$. Both $\lambda_{1}$ and $\lambda_{2}$ are positive since $S_{0}$ is positive definite everywhere. Set $H=1 / c^{2}-1 / d^{2}$. Then the minimum eigenvalue at $y$ is greater than or equal to $H / \lambda_{1}$ and the minimum eigenvalue at $z$ is greater than or equal to $H / \lambda_{2}$. Note that since $1 /\left(4 c^{2}\right) \leq 1 / d^{2}$ we have

$$
H \geq 3 / d^{2} .
$$




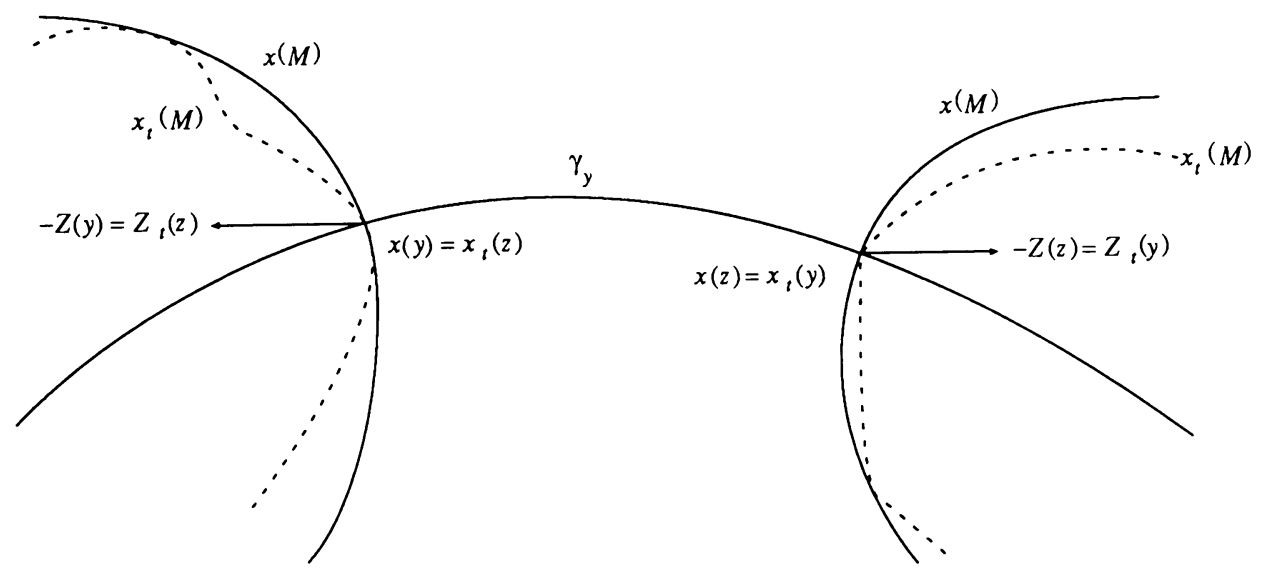

Figure 1

By the minimality of $t$, the image of $x_{t}$ near $z$ must lie on the side of $x(M)$ determined by $Z_{t}(y)=-Z(z)$, as Figure 1 indicates.

Thus,

$$
\left(1 /\left|V_{i}^{2}\right|\right) S\left(V_{i}, V_{i}\right) \leq-H / \lambda_{i}, \quad i=1,2,
$$

for all $V_{1}$ tangent to $x_{t}$ at $y$ and for all $V_{2}$ tangent to $x_{t}$ at $z$. In particular, let $V_{i}(s)$ be the $M$-Jacobi field along the geodesic $\gamma_{t}$ given by

$$
V_{i}(s)=\left(x_{s}\right)_{*} V_{i}, \quad i=1,2,
$$

where $V_{1}$ is a unit vector corresponding to the maximal eigenvalue, $\lambda_{1}$, of $S_{0}$ at $y$ and $V_{2}$ is a unit vector corresponding to the maximal eigenvalue, $\lambda_{2}$, of $S_{0}$ at $z$. Then we have, for $i, j=1,2$ and $i \neq j$,

$$
\left(1 /\left|V_{i}(t)\right|\right) D \mid d s\left(\left|V_{i}(t)\right|\right)=\left(1 /\left|V_{i}(t)\right|^{2}\right) S\left(V_{i}(t), V_{i}(t)\right) \leq-H / \lambda_{j},
$$

By Lemma 4 , for $i, j=1,2$ and $i \neq j$, we have

$$
\left(1 /\left|V_{i}\right|(t)\right)\left(\lambda_{i} \cos (t / d)-(1 / d) \sin (t / d)\right) \leq-H / \lambda_{j} .
$$

Since $t<\frac{1}{2} \pi d$, we conclude that

$$
\sin (t / d) /\left(\left|V_{i}(t)\right| d\right)>H / \lambda_{j} .
$$

Also by Lemma 4, for $i=1,2$,

$$
\left|V_{i}(t)\right| \geq d \lambda_{i} \sin (t / d)+\cos (t / d)>d \lambda_{i} \sin (t / d) .
$$

Combining formulas (2) and (3) gives $1 /\left(d^{2} \lambda_{1}\right)>H / \lambda_{2}$ and $1 /\left(d^{2} \lambda_{2}\right)>H / \lambda_{1}$. Thus by (1), we have $\lambda_{1} \geq 3 \lambda_{2}$ and $\lambda_{2} \geq 3 \lambda_{1}$, which is impossible since $\lambda_{1}$ and $\lambda_{2}$ are positive. Hence $x$ must be an imbedding.

Since $M$ is diffeomorphic to an $(n-1)$-sphere and is imbedded by $x$ in the interior of $B(p, R)$, then $x(M)$ bounds a well-defined interior, Int $x(M)$, by the generalized Jordan-Brouwer Separation Theorem [11, vol. 1, p. 591]. 
Furthermore, $B(p, R)$ is strongly convex. Hence we need only to show that the unique minimal geodesic joining any two points of Int $x(M)$ lies in Int $x(M)$.

The following proof, which is included for completeness, is well known and is attributed to Erhard Schmidt [8, p. 241]. Let $y$ and $z$ be two points in Int $x(M)$ and let $\gamma$ be the geodesic segment joining them. Suppose $\gamma$ intersects $x(M)$. Since Int $x(M)$ is connected, there is a path $\alpha(t), 0 \leq t \leq a$, from $y$ to $z$ entirely in Int $x(M)$. Let $\beta_{t}$ be the geodesic segment from $y$ to $\alpha(t)$. There is a smallest $t$ such that $\beta_{t}$ intersects $x(M)$, say $t=b$. Let $q$ be the first point of $\beta_{b}$ which lies in $x(M)$. Since $b$ is the smallest possible, $\beta_{b}$ must lie in $x(M) \cup \operatorname{Int} x(M)$ and $\beta_{b}$ must be tangent to $x(M)$ at $q$. However, since $S$ is positive definite at $q$, any geodesic segment which is tangent to $x(M)$ at $q$ locally lies outside of $x(M)$. This contradiction shows that $\gamma$ must lie in Int $x(M)$, and hence that $x(M)$ bounds a convex body. This completes the proof of Theorem 2 .

\section{TightNesS OF BOUNDS}

Finally we shall verify that the bound $R<\frac{1}{2} \pi c$, given in Theorem 1 , is the best possible. Let $N$ be a simply connected space of constant curvature less than or equal to $1 /\left(4 c^{2}\right)$. For each $\varepsilon>0$, we construct a surface $M$, isometrically imbedded into $N$, such that $K(M) \geq 1 / c^{2}$ and $M$ lies in no $N$-balls of radius $\pi c-\varepsilon$.

If $N$ is a Euclidean $n$-space it is well known that there is a family of incomplete surfaces of revolutions with curvature $1 / c^{2}$ whose extrinsic diameters approach $\pi c$. By rounding the corners, one achieves a family of surfaces with curvature at least $1 / c^{2}$.

If $N$ has constant curvature $1 / d^{2}$, with $1 / d^{2} \leq 1 /\left(4 c^{2}\right)$, or constant curvature $-1 / d^{2}$, then a family of surfaces of rotation can be generated in a similar manner. In a 2-dimensional totally geodesic submanifold of $N$, let $\gamma$ be a geodesic. Choose a point $p$ on $\gamma$ and a parallel unit normal field $V$ along $\gamma$. An orthogonal grid is formed by the geodesics tangent to $V$ and the curves $\gamma_{r}$ formed by the points of distance $r$ along these geodesics. Let $g$ represent the distance along $\gamma$ and $r$ the distance perpendicular to $\gamma$.

For $0<a \leq c$ and $-\frac{1}{2} \pi c \leq s \leq \frac{1}{2} \pi c$, choose $r_{a}(s)$ and $g_{a}(s)$ to satisfy the equations

$$
\begin{gathered}
d \cdot \sin \left(r_{a}(s) / d\right)=a \cdot \cos (s / c), \\
g_{a}(s)=\int_{0}^{s}\left(1 / \cos \left(r_{a} / d\right)\right)\left(1-\left(d r_{a} / d t\right)^{2}\right)^{1 / 2} d t
\end{gathered}
$$

if $K(N)=1 / d^{2}$; or the equations

$$
\begin{gathered}
d \sinh \left(r_{a}(s) / d\right)=a \cdot \cos (s / c), \\
g_{a}(s)=\int_{0}^{s}\left(1 / \cosh \left(r_{a} / d\right)\right)\left(1-\left(d r_{a} / d t\right)^{2}\right)^{1 / 2} d t
\end{gathered}
$$


if $K(N)=-1 / d^{2}$. Let $\alpha_{a}$ be the curve given in the $(g, r)$-coordinate system by $\alpha_{a}(s)=\left(g(s), r_{a}(s)\right)$. Calculations show that the integrals are well defined, $g_{a}(s)$ is a regular increasing function of $s$, and that $s$ is the arclength parameter of $\alpha_{a}$.

The map $s \rightarrow \alpha_{a}(s)$ is regular and one-to-one since there are no focal points of $\gamma$ along any geodesic normal to within distance $r(s)$ of $\gamma$. Thus a surface of rotation, $M_{a}$, is generated in $N$ when $\alpha_{a}$ is rotated about $\gamma$ in some 3dimensional totally geodesic submanifold. Calculations show that these surfaces have constant curvature $1 / c^{2}$, except at the endpoints of $\alpha_{a}$. The surfaces of rotation $M_{a}$ approach $\gamma$ as $a$ approaches 0 . Thus, by rounding the surfaces at the endpoints of $\alpha_{a}$ we have a family of surfaces whose extrinsic diameters approach $\pi c$ and whose sectional curvatures are at least $1 / c^{2}$, as desired.

Note that the restriction $i(N) \geq \pi c$ is necessary in Theorem 2 . The conclusion there is that an isometrically imbedded hypersurface $M$, with $K(M) \geq$ $1 / c^{2}$, bounds a convex body of $N$. Consider our example in $R^{n}$ with the surfaces rotated about the $x$-axis, and identify $x$ with $x+2 k(\pi c-\varepsilon), k$ an integer. The resulting flat manifold has injectivity radius $\pi c-\varepsilon$. A surface constructed as above whose extrinsic diameter exceeds $\pi c-\varepsilon$ will not bound a convex body in the flat manifold since the shortest geodesic joining a pair of points near the ends of the diameter lies outside the body which the surface bounds.

As has already been mentioned, do Carmo and Warner showed that an isometric immersion $x$ of an $(n-1)$-manifold $M$, with $K(M) \geq 1 / c^{2}$, into a sphere of curvature $1 / c^{2}$ is an imbedding and that either $x(M)$ is totally geodesic or it bounds a convex body in $N$. So the question still remains whether or not our restriction $K(N) \leq 1 /\left(4 c^{2}\right)$ can be improved in general. Furthermore, if $x(M)$ is not required to bound a convex body, one can ask whether $i(N) \geq \pi c$ can be replaced by $i(N)>\frac{1}{2} \pi c$ or some other restriction.

\section{REFERENCES}

1. S. Alexander, Locally convex hypersurfaces of negatively curved spaces. Proc. Amer. Math. Soc. 64 (1977), 321-325.

2. R. L. Bishop and R. J. Crittenden, Geometry of manifolds. Academic Press. New York. 1964.

3. J. Cheeger and D. Ebin, Comparison theorems in Riemannian geometry: North-Holland. New York, 1975.

4. M. P. do Carmo and E. Lima, Immersons of manifolds with nonnegative sectional curvatures. Bol. Soc. Brasil. Mat. 2 (1972), 9-22.

5. M. P. do Carmo and F. W. Warner. Rigidity and convexity of hypersurfaces in spheres, J. Differential Geom. 4 (1970), 133-144.

6. S. S. Chern and R. K. Lashof, On the total curvature of immersed manifolds. Amer. J. Math. 79 (1957), 306-318.

7. J. Hadamard, Sur certaines proprietes des trajectories en d!namique. J. Math. Pures Appl. 3 (1897), 331-387. 
8. J. van Heijenoort, On locally convex manifolds, Comm. Pure Appl. Math. 5 (1952), 223242.

9. H. Karcher, Riemannian center of mass and mollifier smoothing, Comm. Pure Appl. Math. 30 (1977), 509-541.

10. R. Sacksteder, On hypersurfaces with no negative sectional curvatures, Amer. J. Math. 82 (1960), 609-630.

11. M. Spivak, A comprehensive introduction to differential geometry, Publish or Perish, Berkeley, Calif., 1979.

12. J. Spruck, On the radius of the smallest ball containing a compact manifold of positive curvature, J. Differential Geom. 8 (1973), 257-258.

13. J. J. Stoker, Uber die gestalt der positiv gekrummten offenen Flache, Compositio Math. 3 (1936), 55-88.

14. V. A. Toponogov, Riemannian spaces having their curvature bounded below by a positive number, Amer. Math. Soc. Transl. 37 (1964), 291-336.

15. I. Tribuzy, Convex immersions into positively-curved manifolds, Bol. Soc. Brasil. Mat. 17 (1986), 21-39.

16. F. W. Warner, Extension of the Rauch comparison theorem to submanifolds, Trans. Amer. Math. Soc. 122 (1966), 341-356.

17. H. Wu, The spherical images of convex hypersurfaces, J. Differential Geom. 9 (1974), 279 290.

Department of Mathematics, University of Illinois, Urbana, Illinois 61801

Current address: 1421 A Galaxy Avenue, Scott AFB, Illinois 62225 